\title{
Correlation of allograft size versus body mass index to the incidence of hyperfiltration injury among kidney transplant recipients
}

\author{
Carl Davin Tam, Dennis Serrano
}

Department of Organ Transplantation and Vascular Surgery, National Kidney and Transplant Institute, Quezon City, Philippines

Background: Mismatch of donor and recipient factors, particular renal size relative to recipient body mass index, were identified as risk factors of hyperfiltration injury. This study correlated the allograft size versus body mass index (BMI) to the incidence of hyperfiltration injury.

Methods: A retrospective records analysis of 150 transplant recipients. Kidney volume was computed based on computed tomography angiogram using the ellipsoidal formula, which was then correlated with recipients BMI. The observation points from the time of kidney transplantation, was on the day of discharge, at 3 months and one year after transplantation with estimated glomerular filtration rate, serum creatinine, and proteinuria as surrogate markers for hyperfiltration injury. Pearson correlation and Kendall's tau-b correlation were used to determine the relationship of the BMI and kidney volume.

Results: The 150 kidney transplant patients has a mean age of 30.7 years (range, $18-60$ years) with male predominance (66\% vs. $34 \%)$. There was no sufficient evidence to show the correlation of kidney volume and BMI on discharge, at 3 months and 1 year follow-up with Kendall's tau-b correlation of 0.008 ( $P=0.910),-0.422(P=0.082)$, and $-0.502(P=0.049)$, respectively.

Conclusions: The findings suggested that kidney size and recipients BMI are independent factors in determining kidney function up to 1 year follow-up. However, contradicting to other literature, a more longer follow-up may be needed before a direct conclusions be made.

Corresponding author: Carl Davin Tam

E-mail: organ.transplant@nkti.gov.ph

(c) The Korean Society for Transplantation

This is an Open Access article distributed under the terms of the Creative Commons Attribution Non-Commercial License (http://creativecommons.org/licenses/by-nc/4.0/) which permits unrestricted non-commercial use, distribution, and reproduction in any medium, provided the original work is properly cited. 